Research Article

\title{
Synchronization of a Dual-Mass Vibrating System with Two Exciters
}

\author{
Yunshan Liu $\mathbb{D}^{1},{ }^{1,2}$ Xueliang Zhang $\mathbb{D}^{1},{ }^{1}$ Dawei Gu, ${ }^{1}$ Lei Jia $\left(\mathbb{D},{ }^{1}\right.$ and Bangchun Wen ${ }^{1}$ \\ ${ }^{1}$ School of Mechanical Engineering and Automation, Northeastern University, 110819 Shenyang, China \\ ${ }^{2}$ Guidaojiaotong Polytechnic Institute, 110023 Shenyang, China \\ Correspondence should be addressed to Yunshan Liu; liuyunshan75@163.com
}

Received 21 March 2019; Revised 20 August 2019; Accepted 24 December 2019; Published 21 January 2020

Academic Editor: Huu-Tai Thai

Copyright (c) 2020 Yunshan Liu et al. This is an open access article distributed under the Creative Commons Attribution License, which permits unrestricted use, distribution, and reproduction in any medium, provided the original work is properly cited.

From the perspective of theoretical derivation, numerical simulation, and engineering application, the vibratory synchronization characteristics of a dual-mass vibrating system driven by two exciters, were studied. The differential motion equations of the total system were calculated using Lagrange's equations, and the responses of the vibrating system in the steady state were derived by Laplace transform. The synchronization criterion between two exciters was deduced by using the averaging method. Based on the Hamilton principle, the stability criterion of the vibrating system in synchronous states is given. According to the theoretical results, the coupling characteristics between two exciters such as synchronization and stability were analyzed numerically. Some analyses of the numerical simulation of the system were carried out, which fully support the theoretical results. The rotational speed of two exciters, their phase difference, responses, and difference of responses of two rigid bodies were studied quantitatively in the subresonant state and super-resonant state of the system. This paper presents a practical example of vibratory synchronization of a dual-mass system driven by two exciters in engineering.

\section{Introduction}

Synchronization phenomena exist in many aspects of life, such as synchronization in musical instruments, gears, and self-sustained electromechanical devices [1-3]. Huygens [4] first discovered the vibratory synchronization phenomenon in a mechanical system.

In the 1960s, Blekhman [5-7] proposed a synchronization theory for mechanical exciters. The application of this theory made the work of synchronous machines extremely simple, making the maintenance easy and convenient and significantly improving the reliability of these machines. Thus, synchronization technology has been widely applied in engineering.

From 1975 to 1985 , Wen et al. $[8,9]$ made significant progress in the field of designing vibrating machines using the averaging method of small parameters. Babitsky and Krupenin [10] used the averaging method in combination with the single-frequency method to yield useful formulae for utilizing particular vibrating systems. Inoue et al. $[11,12]$ reported their study results on three-time frequency synchronization of a dual-motor vibrating machine. Zhao et al. [13] applied the Lyapunov function to derive the stability condition of synchronization. Based on previous methods, Zhang et al. [14-16] studied and analyzed synchronization problems of two or more than two exciters in a single-mass vibrating system. Balthazar et al. $[17,18]$ gave some short comments on the synchronization of exciters on a flexible portal frame structure. Nijmeijer and Alejandro [19] proved analytically and validated experimentally that synchronization can be achieved in multicomposed mechanical systems. Kong et al. [20] studied the controlled synchronization of vibrating machines driven by AC motors. Synchronization of a dualmass vibrating screen has the advantages of a simple structure, easy installation and maintenance, low energy 
consumption, and good vibration isolation performance. However, the synchronization of a dual-mass system with two exciters has been rarely considered.

In this paper, a theoretical, numerical, and engineering application study was conducted on the synchronization of a vibrating system with dual mass driven by two exciters. In Section 2, the dynamic model of a considered vibrating system is introduced, and the differential motion equations of the total system are given using Lagrange's equations. In Section 3, the synchronization and stability criterion of synchronous states are deduced. In Section 4, based on the above theoretical results, numerical analyses are presented for coupling dynamic characteristics between two exciters. In Section 5, simulation analyses on the subresonant case and the super-resonant case of the vibrating system are conducted. In Section 6, an engineering application example, which was designed by the synchronization theory and the dynamic model of the dual-mass vibrating system with two exciters in this paper, is introduced. Finally, some conclusions are provided.

Based on the ratio between operating frequency and natural frequency of the main vibrating system (i.e, $\left.z=\omega_{m 0} / \omega_{0} \approx \omega_{m 0} / \omega_{\text {Inv }}^{\prime}\right)$, the total system of vibrating machines can be classified into five types of resonance: subresonant $(z<0.95)$, near-resonant $(0.95 \leq z \leq 1.05)$, nearsubresonant $(0.95 \leq z \leq 1)$, near-super-resonant $(1 \leq z \leq 1.05)$, and super-resonant $(z>1.05)$ vibrating systems. Additionally, the succeeding parts also conducted some quantitative analyses of synchronization according to the above classification.

\section{Mathematical Modeling of the Vibrating System}

Figure 1 shows the dynamic model of a vibrating system with dual mass driven by two exciters, including the upper mass $m_{1}$ with two exciters and the lower mass $m_{2}$. The exciters consist of unbalanced rotors driven by induction motors

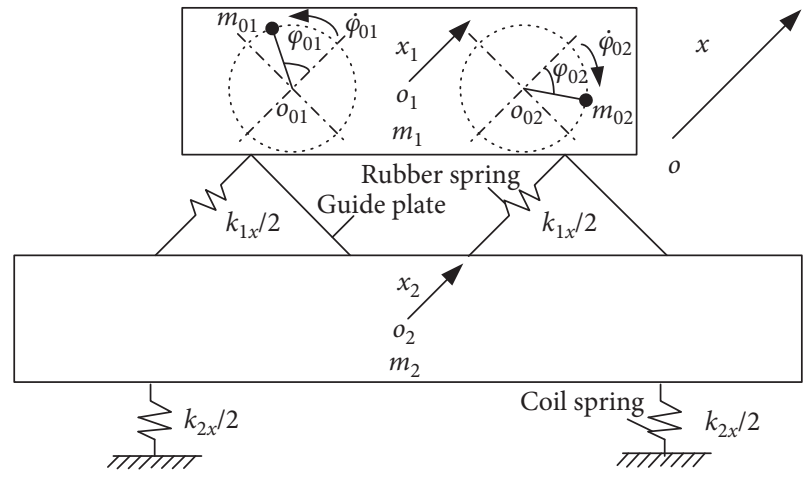

FIgURE 1: Dynamic model of a vibrating system with dual mass driven by two exciters.

separately. The two exciters are assumed to be identical. The two masses $m_{1}$ and $m_{2}$ connected via shear rubber springs $k_{1 x}$ and guide plates are assumed to be rigid bodies. The rigid body $m_{2}$ is linked to the foundation by coil springs $k_{2 x}$. Compared with the shear rubber springs $k_{1 x}$, the coil springs $k_{2 x}$ are very small, i.e., $k_{2 x}<<k_{1 x}$. All the springs are assumed to be linearly elastic. The two exciters installed on the rigid body $m_{1}$ are driven by two induction motors rotating in opposite directions. Because of the limiting role of guide plates, the movement of the entire system is assumed to be plane motion in the $x$-direction. $x_{i}$ $(i=1,2)$ is the response of rigid body $m_{i}(i=1,2)$ in the $x$ direction.

2.1. Differential Equations of Absolute Motion of the Vibrating System. By selecting $x_{1}, x_{2}, \varphi_{01}$, and $\varphi_{02}$ as the generalized coordinates and using Lagrange's equations, the differential motion equations of the total system can be expressed as [14]

$$
\begin{aligned}
M_{1} \ddot{x}_{1}+f_{1 x}\left(\dot{x}_{1}-\dot{x}_{2}\right)+k_{1 x}\left(x_{1}-x_{2}\right) & =\sum_{i=1}^{2} m_{0 i} r\left(\dot{\varphi}_{0 i}^{2} \sin \varphi_{0 i}-\ddot{\varphi}_{0 i} \cos \varphi_{0 i}\right), \\
M_{2} \ddot{x}_{2}+f_{2 x} \dot{x}_{2}+f_{1 x}\left(\dot{x}_{2}-\dot{x}_{1}\right)+k_{2 x} x_{2}+k_{1 x}\left(x_{2}-x_{1}\right) & =0, \\
J_{01} \ddot{\varphi}_{01}+f_{01} \dot{\varphi}_{01} & =T_{e 1}-m_{01} r \ddot{x}_{2} \cos \varphi_{01}, \\
J_{02} \ddot{\varphi}_{02}+f_{02} \dot{\varphi}_{02} & =T_{e 2}-m_{02} r \ddot{x}_{2} \cos \varphi_{02},
\end{aligned}
$$

with

$$
\begin{aligned}
M_{1} & =m_{1}+m_{01}+m_{02}, \\
M_{2} & =m_{2}, \\
J_{0 i} & =m_{0 i} r^{2}=J_{0}, \\
m_{01} & =m_{02}=m_{0},
\end{aligned}
$$

$$
i=1,2 \text {, }
$$

where $k_{i x}$ is the spring stiffness in $x$-direction, $i=1,2 ; r$ is the eccentric radius of exciter; $J_{0 i}$ is the moment of inertia of the exciter $i i=1,2 ; f_{i x}$ is the damping constant in $x$-direction, $i=1,2 ; m_{0 i}$ is the mass of exciter $i, i=1,2 ; m_{i}$ is the mass of rigid body $m_{i}, i=1,2 ; \varphi_{0 i}$ is the phase of exciter $i$, $i=1,2 ; T_{e i}$ is the electromagnetic torque of exciter $i, i=1,2$; $f_{0 i}$ is the damping coefficient of the shaft of induction motor $i, i=1,2 ;(\bullet)$ represents $\mathrm{d} \bullet / \mathrm{d} t ;(\bullet)$ represents $\mathrm{d}^{2} \bullet / \mathrm{d} t^{2} ; x_{i}$ is the displacement of rigid body $m_{i}(i=1,2)$ in $x$-direction; $\dot{x}_{i}$ 
is the velocity of rigid body $m_{i}(i=1,2)$ in $x$-direction; and $\ddot{x}_{i}$ is the acceleration of rigid body $m_{i}(i=1,2)$ in $x$-direction.

2.2. Differential Equations of Relative Motion of the System. Because the system includes two masses, it is necessary to investigate their relative motion as follows.

For applications in engineering, the exciters used in synchronous states in a vibrating system are usually identical. Therefore, the masses of the two exciters are assumed to be the same. Here, $m_{0}$ is the mass of the standard exciter, i.e., $m_{01}=m_{02}=m_{0}$. In addition, the average phase of two exciters and their phase difference are $\varphi$ and $2 \alpha$, respectively:

$$
\begin{aligned}
& \varphi_{01}=\varphi+\alpha, \\
& \varphi_{02}=\varphi-\alpha .
\end{aligned}
$$

Assuming that two exciters can operate synchronously and their average angular velocity is $\omega_{\text {Inv }} \approx \omega_{0}$, i.e. $\dot{\varphi}=\omega_{m 0}$, based on $[8,9]$, the relationship between displacements and accelerations in the steady state can be described as follows:

$$
\begin{aligned}
& \ddot{x}_{1}=-\omega_{m 0}^{2} x_{1}, \\
& \ddot{x}_{2}=-\omega_{m 0}^{2} x_{2} .
\end{aligned}
$$

During the steady state, the angular accelerations of two exciters are close to zero and can thus be ignored. Substituting equation (4) into the first two differential formulae in equation (1), and considering $k_{2 x} \ll k_{1 x}[8,9]$,

$$
\begin{aligned}
& M_{1} \ddot{x}_{1}+f_{1 x}\left(\dot{x}_{1}-\dot{x}_{2}\right)+k_{1 x}\left(x_{1}-x_{2}\right)=2 m_{0} r \omega_{m 0}^{2} \sin \varphi \cos \alpha, \\
& M_{2} \ddot{x}_{2}-f_{1 x}\left(\dot{x}_{1}-\dot{x}_{2}\right)-k_{1 x}\left(x_{1}-x_{2}\right)=0 .
\end{aligned}
$$

Combining equation (3) and considering $\dot{\varphi}=\omega_{m 0}$ in the steady state, the differential equation of relative motion between rigid bodies $m_{1}$ and $m_{2}$ in the $x$-direction can be expressed as follows:

$$
m \ddot{x}_{12}+f_{1 x} \dot{x}_{12}+k_{1 x} x_{12}=\frac{2 M_{1}}{M_{1}+M_{2}} m_{0} r \omega_{m 0}^{2} \sin \varphi \cos \alpha,
$$

with

$$
\begin{aligned}
m & =\frac{M_{1} M_{2}}{M_{1}+M_{2}}, \\
\ddot{x}_{12} & =\ddot{x}_{1}-\ddot{x}_{2}, \\
\dot{x}_{12} & =\dot{x}_{1}-\dot{x}_{2}, \\
x_{12} & =x_{1}-x_{2},
\end{aligned}
$$

where $m$ is the induced mass of the entire system; $x_{12}, \dot{x}_{12}$, and $\ddot{x}_{12}$ are the relative displacement, relative velocity, and relative acceleration between rigid bodies $m_{1}$ and $m_{2}$ in the $x$-direction.

By considering equation (6), the natural frequency of relative motion between two rigid bodies in the $x$-direction can be obtained. This is often viewed as the natural frequency of the main vibrating system:

$$
\omega_{0}=\sqrt{\frac{k_{1 x}}{m}} .
$$

The response of relative motion between two rigid bodies can be rewritten as follows:

$$
x_{12}=A_{12} \sin \left(\varphi-\gamma_{12}\right) \cos \alpha
$$

with

$$
\begin{aligned}
A_{12} & =\frac{2 z_{0}^{2} r m_{0}}{M_{1} \sqrt{1+z_{0}^{4}+2 z_{0}^{2}\left(2 \xi_{1 x}^{\prime 2}-1\right)}} \\
\gamma_{12} & =\arctan \left(\frac{2 \xi_{1 x}^{\prime} z_{0}}{1-z_{0}^{2}}\right) \\
z_{0} & =\frac{\omega_{m 0}}{\omega_{0}} .
\end{aligned}
$$

For small dampers in engineering, $f_{1 x}=2 \xi_{1 x}^{\prime} m \omega_{0}$, where $\xi_{1 x}^{\prime}$ is the equivalent relative damping coefficient, $\xi_{1 x}^{\prime} \leq 0.07$ [8].

When $z_{0}=1$ (i.e., $\omega_{m 0}=\omega_{0}$ ), the maximum of $A_{12}$ in equation (8) can be obtained at the resonant point. This denotes that $\omega_{0}$ is the natural frequency of relative motion with the opposite phases of two rigid bodies in the $x$ direction.

According to the mathematical solution of the extremum principle [21], the amplitude of response of relative motion with the inverse phases can be expressed as follows:

$$
\lambda_{12}=\left|A_{12} \sin \left[\alpha+\arctan \left(\frac{\cos 2 \alpha+1}{\sin 2 \alpha}\right)\right] \cos \alpha\right| \text {. }
$$

$\lambda_{12}$ is important in engineering applications, and the graph based on (11) is described in Section 4.

\section{Synchronization Analysis of Two Exciters}

For the first two formulae of equation (1), the transfer function method is used, and this approach is based on the Laplace transform. By combining with equation (3), some responses of the vibrating system can be expressed as follows:

$$
\begin{aligned}
& x_{1}=2 m_{0} r \omega_{m 0}^{2} \sqrt{\frac{c_{1}^{2}+d_{1}^{2}}{a_{1}^{2}+b_{1}^{2}}} \sin \left(\varphi-\gamma_{1 x}\right) \cos \alpha, \\
& x_{2}=2 m_{0} r \omega_{m 0}^{2} \sqrt{\frac{g_{1}^{2}+e_{1}^{2}}{a_{1}^{2}+b_{1}^{2}}} \sin \left(\varphi-\gamma_{2 x}\right) \cos \alpha,
\end{aligned}
$$

with 


$$
\begin{aligned}
& a_{1}=\omega_{m 0}^{4} M_{1} M_{2}-\omega_{m 0}^{2}\left(M_{1} k_{1 x}+M_{1} k_{2 x}\right. \\
& \left.+f_{1 x} f_{2 x}+k_{1 x} M_{2}\right)+k_{1 x} k_{2 x} \\
& b_{1}=-\omega_{m 0}^{3}\left(f_{2 x} M_{2}+f_{1 x} M_{1}+f_{2 x} M\right) \\
& +\omega_{m 0}\left(f_{1 x} k_{2 x}+f_{2 x} k_{1 x}\right) f_{1 x} k_{2 x}+\omega_{m 0} f_{2 x} k_{1 x}, \\
& c_{1}=k_{1 x} \text {, } \\
& d_{1}=\omega_{m 0} f_{1 x} \text {, } \\
& g_{1}=k_{1 x}+k_{2 x}-M_{1} \omega_{m 0}^{2} \text {, } \\
& e_{1}=f_{1 x} \omega_{m 0}+f_{2 x} \omega_{m 0}, \\
& \gamma_{1 x}= \begin{cases}\arctan \left(\frac{b_{1} c_{1}-a_{1} d_{1}}{a_{1} c_{1}+b_{1} d_{1}}\right), & a_{1} c_{1}+b_{1} d_{1}>0, \\
\pi+\arctan \left(\frac{b_{1} c_{1}-a_{1} d_{1}}{a_{1} c_{1}+b_{1} d_{1}}\right), & a_{1} c_{1}+b_{1} d_{1}<0,\end{cases} \\
& \gamma_{2 x}= \begin{cases}\arctan \left(\frac{b_{1} g_{1}-a_{1} e_{1}}{a_{1} g_{1}+b_{1} e_{1}}\right), & a_{1} g_{1}+b_{1} e_{1}>0, \\
\pi+\arctan \left(\frac{b_{1} g_{1}-a_{1} e_{1}}{a_{1} g_{1}+b_{1} e_{1}}\right), & a_{1} g_{1}+b_{1} e_{1}<0,\end{cases}
\end{aligned}
$$

where $\gamma_{1 x}$ is the phase angle of rigid body $m_{1}$ in the $x$-direction; $\gamma_{2 x}$ is the phase angle of rigid body $m_{2}$ in the $x$ direction.

3.1. Synchronization Criterion of Two Exciters. According to equation (1), the coupling matrix of inertia and stiffness, as well as the characteristic equation of two rigid bodies in the $x$-direction can be expressed as follows:

$$
\begin{aligned}
M & =\left(\begin{array}{cc}
M_{1} & 0 \\
0 & M_{2}
\end{array}\right), \\
K & =\left(\begin{array}{cc}
k_{1 x} & -k_{1 x} \\
-k_{1 x} & k_{1 x}+k_{2 x}
\end{array}\right), \\
\Delta\left(\omega^{2}\right) & =\left|\begin{array}{cc}
k_{1 x}-\omega^{2} M_{1} & -k_{1 x} \\
-k_{1 x} & k_{1 x}+k_{2 x}-\omega^{2} M_{2}
\end{array}\right|,
\end{aligned}
$$

where $M$ is the inertia and $K$ is the stiffness. They are both coupled matrices; $\Delta\left(\omega^{2}\right)=0$ is the characteristic equation.

Making $\Delta\left(\omega^{2}\right)=0$, i.e.,

$$
\omega^{4} M_{1} M_{2}-\omega^{2}\left(M_{1} k_{1 x}+M_{1} k_{2 x}+M_{2} k_{1 x}\right)+k_{1 x} k_{2 x}=0 .
$$

Based on equation (15), two natural frequencies of the two rigid bodies in the $x$-direction can be obtained as follows:

$$
\begin{gathered}
\omega_{\text {Inv }}=\sqrt{\frac{M_{1}\left(k_{1 x}+k_{2 x}\right)+M_{2} k_{1 x}+\sqrt{M_{1}^{2} k_{1 x}^{2}+M_{1}^{2} k_{2 x}^{2}+M_{2}^{2} k_{1 x}^{2}+2\left(M_{1}^{2} k_{1 x} k_{2 x}-M_{1} M_{2} k_{1 x} k_{2 x}+M_{1} M_{2} k_{1 x}^{2}\right)}}{2 M_{1} M_{2}}}, \\
\omega_{\mathrm{Sa}}=\sqrt{\frac{M_{1}\left(k_{1 x}+k_{2 x}\right)+M_{2} k_{1 x}-\sqrt{M_{1}^{2} k_{1 x}^{2}+M_{1}^{2} k_{2 x}^{2}+M_{2}^{2} k_{1 x}^{2}+2\left(M_{1}^{2} k_{1 x} k_{2 x}-M_{1} M_{2} k_{1 x} k_{2 x}+M_{1} M_{2} k_{1 x}^{2}\right)}}{2 M_{1} M_{2}}} .
\end{gathered}
$$

The stiffness of shear rubber springs $k_{1 x}$ is much larger than that of coil springs $k_{2 x}$, i.e., $k_{2 x} \ll k_{1 x}$; therefore, $k_{2 x}$ can be ignored, and then $\omega_{\text {Inv }} \approx \omega_{0}$ can be obtained. $\omega_{\text {Inv }}$ and $\omega_{\text {Sa }}$ are the natural frequency of inverse phases and the samephase relative motion of two rigid bodies in the $x$-direction, respectively.
Provided that the two exciters can reach a steady synchronous state, $\dot{\varphi}_{0 i}=\omega_{m 0}$. By differentiating to $x_{1}$ in equation (12), we obtain $\ddot{x}_{1}$. By considering equation (3), substituting $\ddot{x}_{1}$ into the last two formulae in equation (1), and then integrating them over $\varphi=0 \sim 2 \pi$, the average equilibrium equations of two exciters can be obtained as follows:

$$
\begin{aligned}
& T_{e 01}-f_{01} \omega_{m 0}=\frac{1}{2} m_{0}^{2} r^{2} \omega_{m 0}^{4} \sqrt{\frac{c_{1}^{2}+d_{1}^{2}}{a_{1}^{2}+b_{1}^{2}}}\left(\sin \gamma_{1 x}+\sin 2 \alpha \cos \gamma_{1 x}+\cos 2 \alpha \sin \gamma_{1 x}\right), \\
& T_{e 02}-f_{02} \omega_{m 0}=\frac{1}{2} m_{0}^{2} r^{2} \omega_{m 0}^{4} \sqrt{\frac{c_{1}^{2}+d_{1}^{2}}{a_{1}^{2}+b_{1}^{2}}}\left(\sin \gamma_{1 x}-\sin 2 \alpha \cos \gamma_{1 x}+\cos 2 \alpha \sin \gamma_{1 x}\right),
\end{aligned}
$$


where $f_{0 i}$ is the damping coefficient of axes of induction motor $i$ and $T_{e 0 i}$ is the electromagnetic torque of motor $i$ at the synchronous angular velocity $\omega_{m 0},(i=1,2)$.

In the above process of integration, compared with the change in $\varphi\left(\dot{\varphi}=\omega_{m 0}\right)$ in regard to time $t$, the change in $2 \alpha$ is very small; therefore, $2 \alpha$ is treated as a slow variable parameter. According to the direct motion separation method [5-7], $2 \alpha$ can be denoted by the middle value of its integration $2 \bar{\alpha}$.

Equations (17) and (18) can be rearranged as follows:

$$
\begin{gathered}
T_{e 01}+T_{e 02}-f_{01} \omega_{m 0}-f_{02} \omega_{m 0}=T_{\text {Load }}, \\
\frac{T_{\mathrm{D}}}{T_{\mathrm{C}}}=\sin (2 \bar{\alpha}), \\
T_{\text {Load }}=m_{0}^{2} r^{2} \omega_{m 0}^{4} \sqrt{\frac{g_{1}^{2}+e_{1}^{2}}{a_{1}^{2}+b_{1}^{2}}}\left(\sin \gamma_{2 x}+\cos 2 \alpha \sin \gamma_{2 x}\right), \\
T_{\mathrm{D}}=T_{R 1}-T_{R 2}, \\
T_{\mathrm{C}}=m_{0}^{2} r^{2} \omega_{m 0}^{4} \sqrt{\frac{g_{1}^{2}+e_{1}^{2}}{a_{1}^{2}+b_{1}^{2}}} \cos \gamma_{2 x}, \\
T_{R 1}=T_{e 01}-f_{01} \omega_{m 0}-\frac{1}{2} m_{0}^{2} r^{2} \omega_{m 0}^{4} \sqrt{\frac{g_{1}^{2}+e_{1}^{2}}{a_{1}^{2}+b_{1}^{2}}} \sin \gamma_{2 x}, \\
T_{R 2}=T_{e 02}-f_{02} \omega_{m 0}-\frac{1}{2} m_{0}^{2} r^{2} \omega_{m 0}^{4} \sqrt{\frac{g_{1}^{2}+e_{1}^{2}}{a_{1}^{2}+b_{1}^{2}}} \sin \gamma_{2 x},
\end{gathered}
$$

where $T_{\text {Load }}$ is the total load of two exciters; $T_{R 1}$ and $T_{R 2}$ are the effective electromagnetic output torque of exciters 1 and 2 , respectively; $T_{D}$ is the difference between effective electromagnetic output torque of two exciters; and $T_{\mathrm{C}}$ is the capture torque.

In equation (20), the synchronization criterion between two exciters can be obtained from $|\sin (2 \alpha)|<1$ as follows:

$$
T_{\mathrm{D}}<T_{\mathrm{C}} .
$$

Equations (19) and (20) are the nonlinear formulae of $2 \bar{\alpha}$ and $\omega_{m 0}$; their solutions can be expressed in terms of $2 \bar{\alpha}_{0}$ and $\omega_{m 0}^{*}$, respectively.

In addition, based on equation (19), it can be obtained as follows:

$$
2 \bar{\alpha}=\arcsin \left(\frac{T_{R 1}-T_{R 2}}{T_{\mathrm{C}}}\right) .
$$

Equation (23) can have stable and unstable solutions. The stable solution is denoted by $2 \bar{\alpha}_{0}^{*}$. Additionally, the stability of the solution is ensured by the stability criterion of the synchronous state, which is provided in detail in the next section.

When the parameters of the two exciters are identical (including the motor model), $T_{R 1}-T_{R 2}=0$. According to equation (23), the phase difference between two exciters can be stabilized at zero or $\pi$. In this manner, it is possible to define the coefficient of synchronization ability between two exciters, i.e., $\zeta$ :

$$
\zeta=\left|\frac{T_{\mathrm{C}}}{T_{\text {Load }}}\right|
$$

From the above expression, it can be observed that $\zeta$ is a nonlinear function of $\omega_{m 0}$; this is affected by the structural parameters of the entire system.

3.2. Stability Criterion of Synchronous States. Generally, there are two solutions for $2 \bar{\alpha}$ in equation (23), one is stable, and the other is unstable. Which one is stable depends on the stability criterion of the synchronous states of the system as follows.

The kinetic energy $(T)$ and potential energy $(V)$ of the entire system can be expressed as follows:

$$
\begin{aligned}
& T=\frac{1}{2}\left(M_{1} \dot{x}_{1}^{2}+M_{2} \dot{x}_{2}^{2}+\sum_{i=1}^{2} J_{0 i} \omega_{m 0}^{2}\right) \\
& V=\frac{1}{2} k_{1 x} x_{1}^{2}+\frac{1}{2} k_{2 x}\left(x_{2}-x_{1}\right)^{2} .
\end{aligned}
$$

Hamilton's average amplitude of motion in a period, denoted as $I$, can be expressed intuitively as follows:

$$
I=\frac{1}{2 \pi} \int_{0}^{2 \pi}(T-V) \mathrm{d} \varphi .
$$

According to Wen et al. [8,9], the solution of stable phase difference $\left(2 \bar{\alpha}_{0}^{*}\right)$ in the synchronous states corresponds to a minimum point of $I$. Therefore, the second-order derivative of $I$ in regard of $2 \alpha$ should be positive:

$$
\left.\frac{\mathrm{d}^{2} I}{\mathrm{~d}(2 \alpha)^{2}}\right|_{2 \alpha=2 \bar{\alpha}_{0}^{*}}>0 .
$$

The stability criterion in equation (27) is given in the following three situations:

(1) Considering the case of damping of the system,

Based on equation (12) and substituting equation (26) into equation (27), the synchronization criterion can be obtained as follows:

$$
\left.\frac{\mathrm{d}^{2} I}{\mathrm{~d}(2 \alpha)^{2}}\right|_{2 \alpha=2 \bar{\alpha}_{0}^{*}}=\frac{2 m_{0}^{2} r^{2} \omega_{m 0}^{4}}{a_{1}^{2}+b_{1}^{2}} H \cos \left(2 \bar{\alpha}_{0}^{*}\right)>0,
$$

with

$$
H \cos \left(2 \bar{\alpha}_{0}^{*}\right)>0
$$

where

$$
\begin{aligned}
H= & -2 c_{1} \sqrt{g_{1}^{2}+e_{1}^{2}} \sqrt{c_{1}^{2}+d_{1}^{2}} \cos \left(\gamma_{1 x}-\gamma_{2 x}\right) \\
& +g_{1}\left(c_{1}^{2}+d_{1}^{2}\right)
\end{aligned}
$$

where $H$ is the coefficient of stability ability.

Under the condition $H>0,2 \bar{\alpha}_{0}^{*}$ should be stabilized in the interval of $(-\pi / 2, \pi / 2)$, otherwise, stabilized in the interval of $(\pi / 2,3 \pi / 2)$. Notably, the two exciters are identical. When the above condition $H>0$ is 
satisfied, $2 \bar{\alpha}_{0}^{*}$ is stabilized in the vicinity of zero. This is useful for engineering. Otherwise, $2 \bar{\alpha}_{0}^{*}$ is stabilized in the vicinity of $\pi$.

(2) Ignoring the damping of the system,

For the vibration in engineering, the damping of vibrating equipment is relatively small and can be ignored. Therefore, equation (29) can be written in a dimensionless form as follows:

$$
\begin{array}{ll}
H_{1} \cos \left(2 \bar{\alpha}_{0}^{*}\right)>0, & z_{1}>1, \\
H_{2} \cos \left(2 \bar{\alpha}_{0}^{*}\right)>0, & z_{1}<1,
\end{array}
$$

with

$$
\begin{aligned}
H_{1} & =\frac{r_{m 1}\left(1-z_{1}^{2}\right)}{\left(z_{\mathrm{Inv}}^{2}-1\right)\left(z_{\mathrm{Sa}}^{2}-1\right)}\left(\frac{z_{\mathrm{Inv}} z_{\mathrm{Sa}}}{z_{1}}\right)^{2}, \\
H_{2} & =\frac{r_{m 1}\left(1-z_{1}^{2}\right)}{\left(z_{\mathrm{Inv}}^{2}-1\right)\left(z_{\mathrm{Sa}}^{2}-1\right)}\left(\frac{z_{\mathrm{Inv}} z_{\mathrm{Sa}}}{z_{1}}\right)^{2} \\
\omega_{1} & =\sqrt{\frac{k_{1 x}}{M_{1}}}, \\
\sigma_{12} & =\sqrt{\frac{k_{1 x}}{M_{2}}}, \\
z_{\text {Inv }} & \left.\left.=\frac{\omega_{m 0}}{\omega_{0}} \approx \frac{z_{\mathrm{Inv}} z_{\mathrm{Sa}}}{z_{1} h_{12}}\right)^{2}\right], \\
z_{\mathrm{Sa}} & =\frac{\omega_{m 0}^{\prime}}{\omega_{\mathrm{Sa}}^{\prime}}, \\
h_{12} & =\frac{\omega_{m 0}}{\sigma_{12}} .
\end{aligned}
$$

(3) For some types of vibrating machines in engineering, In engineering, such as nonlinear vibrating centrifuge and vibrating conveyor, the natural frequency of the system is generally designed within the scope of $0<\omega_{\mathrm{Sa}}<\omega_{1}^{\prime}<\omega_{\text {Inv }}$. Equation (31) can be further simplified:

$$
\begin{aligned}
\left(\omega_{1}^{\prime 2}-\omega_{m 0}^{2}\right)<0,\left(\omega_{m 0}^{2}-\omega_{\text {Inv }}^{2}\right)\left(\omega_{m 0}^{2}-\omega_{\mathrm{Sa}}^{2}\right) & <0, \\
\cos \left(2 \bar{\alpha}_{0}^{*}\right) & >0 .
\end{aligned}
$$

Equation (33) can also be simplified as follows:

$$
\omega_{1}^{\prime}<\omega_{m 0}<\omega_{\text {Inv }}, \quad \cos \left(2 \bar{\alpha}_{0}^{*}\right)>0,
$$

with

$$
\omega_{1}^{\prime}=\sqrt{\frac{k_{1 x}^{\prime}}{M_{1}}} .
$$

Therefore, equation (34) can be used as the analytical expression for the simplest stability criterion in engineering design. Under the circumstances, the stable phase difference of two exciters is close to zero. When two exciters operate in a subresonant or near-subresonant state, this system can satisfy the requirements of engineering.

\section{Numerical Characteristics Based on Theoretical Results}

In this part, some quantitative numerical analyses are given to illustrate the effectiveness of abovementioned theoretical results. The parameters of the system are as follows: $m_{1}=1500 \mathrm{~kg}$, $m_{2}=600 \mathrm{~kg}, m_{0}=10 \mathrm{~kg}, k_{1 x}=2400 \mathrm{kN} / \mathrm{m}, \quad k_{2 x}=100 \mathrm{kN} / \mathrm{m}$, $r=0.15 \mathrm{~m}$, and $f_{1 x}=f_{2 x}=2030 \mathrm{kN} /(\mathrm{m} / \mathrm{s})$. Two motors are identical.

In the following numerical discussion, the parameters of two motors are identical. The phase difference is stabilized at zero or $\pi$, while the stability of synchronous solution is based on the stability criterion.

By changing the stiffness coefficients of the springs and based on equations (8) and (16), the frequency ratio of rotational velocities of the two motors to the natural frequency can be derived, i.e.,

$$
z=\frac{\omega_{m 0}}{\omega_{0}} \approx \frac{\omega_{m 0}}{\omega_{\text {Inv }}} .
$$

4.1. Synchronization Ability of Two Exciters. In Section 3.1, the synchronization mechanism of the system is described. The synchronization between two exciters originates from the coupling dynamic characteristics; therefore, the coefficient of synchronization ability $\xi$ is the index to measure and adjust a load of each motor to implement synchronization. As shown in Figure 2, the two breakpoints of $\xi$ are in $\omega_{m 0}=$ $\omega_{1}^{\prime}$ and $\omega_{m 0}=\omega_{\text {Inv }}$. For the latter, it is in $\omega_{m 0}=\omega_{\text {Inv }}$ or $\omega_{0}$ covibration point. This plays an important role in defining reasonable working points in engineering design.

4.2. Stability Ability of Synchronous States. By substituting the above given structural parameters of the system into the formula of $H$ in (29), the coefficient of stability ability of two exciters can be obtained.

As shown in Figure 3, the highest point of the curve is located at $z=1$, i.e., $\omega_{m 0}=\omega_{\text {Inv }} \approx \omega_{0}=74.56 \mathrm{rad} / \mathrm{s}$. This critical point is known as the resonant point. When the condition $\omega_{\text {Inv }}>\omega_{m 0}>\omega_{1}^{\prime}\left(\omega_{1}^{\prime}=40 \mathrm{rad} / \mathrm{s}\right)$ is satisfied, $H>0$. In this case, the vibrating system can operate in the stable state. Moreover, the higher the value of the curve, the stronger the synchronization stability of the system.

4.3. Stable Phase Difference. Because the two motors have the same parameters, the stable phase difference between the two exciters is stabilized in the neighborhood of zero or $\pi$. 


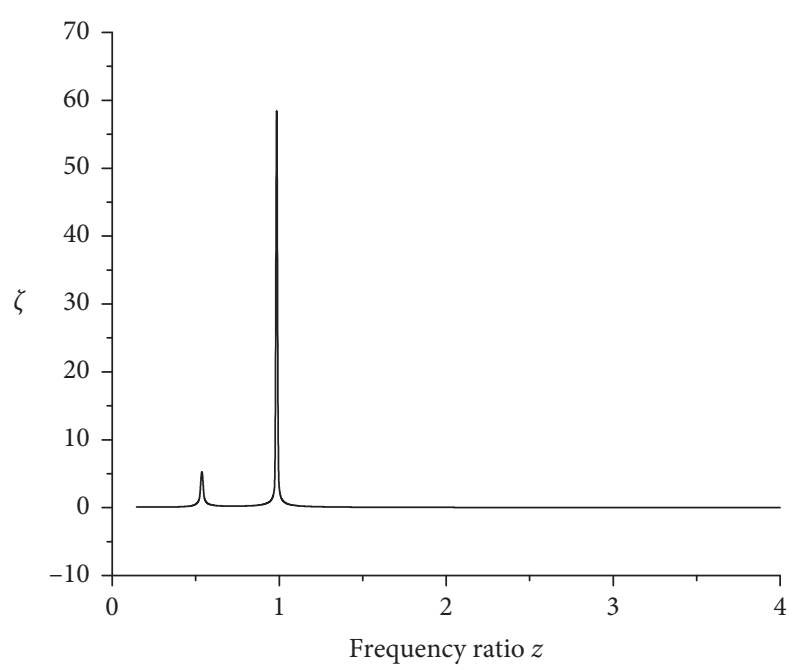

Figure 2: Synchronization ability of two exciters.



FIgURE 3: Ability of stability for two exciters.

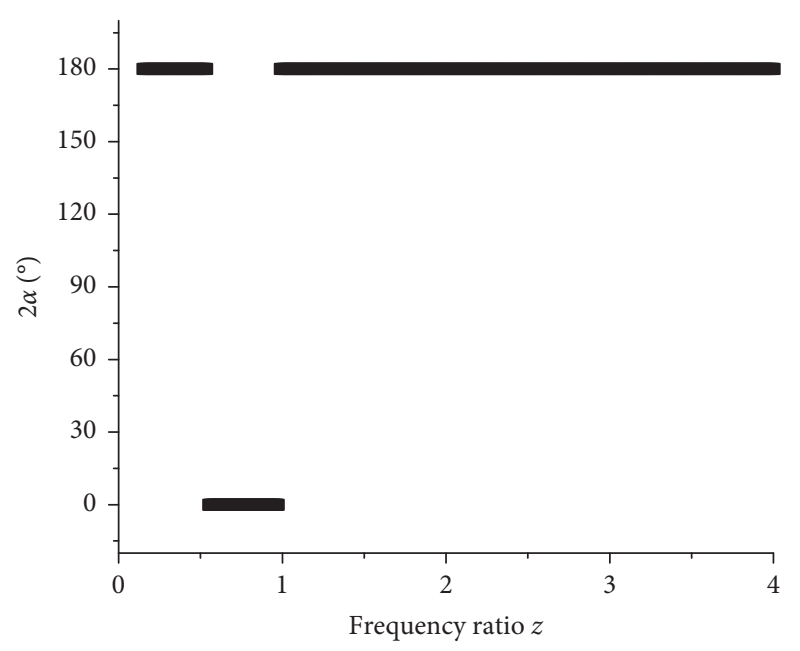

Figure 4: Stable phase difference of two exciters.

Figure 4 is consistent with the theoretical analyses shown in (18). Under the condition $\omega_{1}^{\prime}<\omega_{m 0}<\omega_{\text {Inv }}, H>0$. In this case, the stable phase difference of two exciters is close to zero.

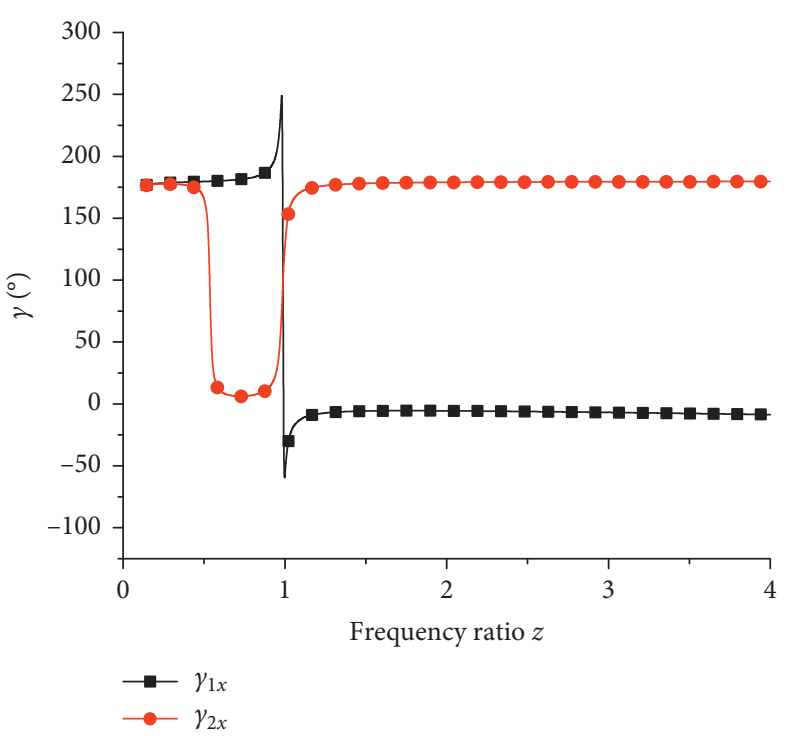

Figure 5: Phase lag angle $\gamma$.

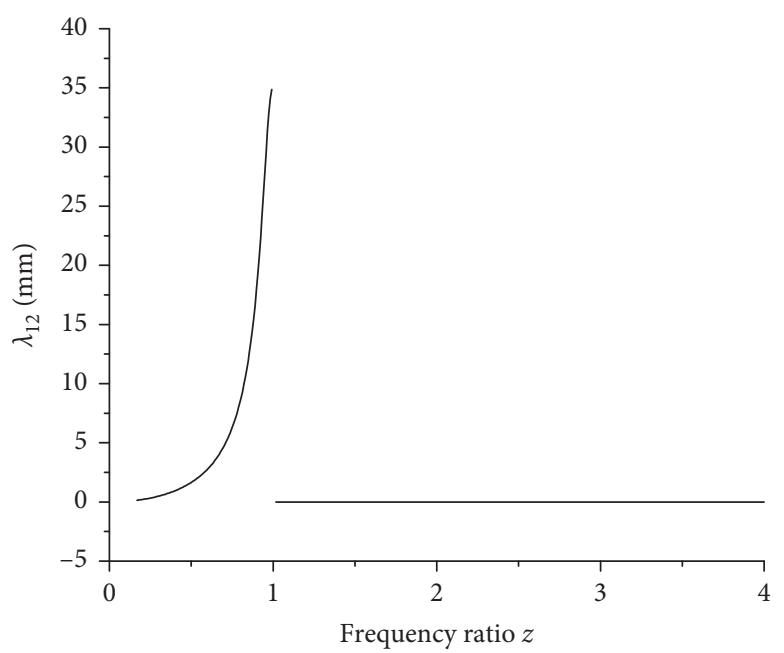

FIgURE 6: Frequency-amplitude relationship.

When the condition $\omega_{m 0}>\omega_{\text {Inv }}$ or $\omega_{m 0}<\omega_{1}^{\prime}$ is satisfied, $H<0$. The stable phase difference is close to $\pi$.

4.4. Phase Angles of Dual Mass. By putting the parameters of the system into $\gamma_{1 x}$ and $\gamma_{2 x}$ in (12), the results are shown in Figure 5. When $\omega_{1}^{\prime}<\omega_{m 0}<\omega_{\text {Inv }}$ and $\omega_{m 0}>\omega_{\text {Inv }}$, the phase angle between rigid body $m_{1}$ and rigid body $m_{2}$ is $\pi$, i.e., $\left|\gamma_{1 x}-\gamma_{2 x}\right|=\pi$. In other words, the two rigid bodies move in opposite directions. This condition is desired in engineering applications.

4.5. Amplitude-Frequency Curve. By introducing the structural parameters of given system into (11), the curve of $\lambda_{12}$ can be obtained. The relationship between $\lambda_{12}$ and frequency ratio $z$ is plotted in Figure 6. (11) shows that the amplitude of response of relative motion with the inverse phases between two rigid bodies $\left(\lambda_{12}\right)$ can be determined 




- - Motor 1

(a)

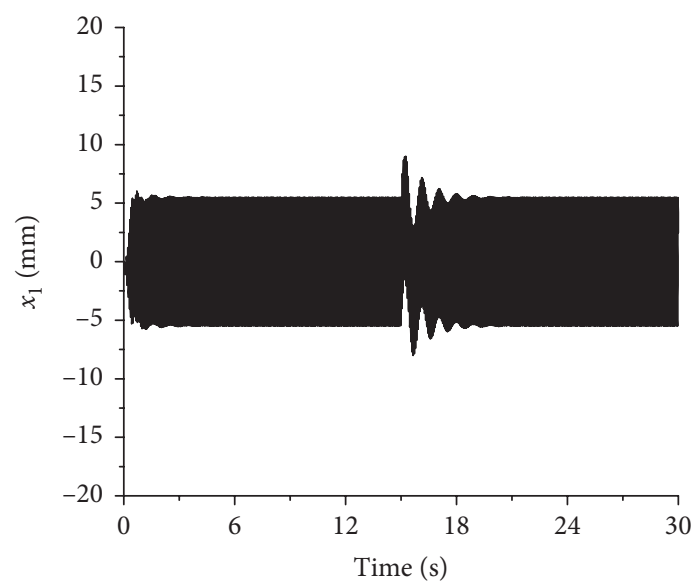

(c)



(b)



(d)

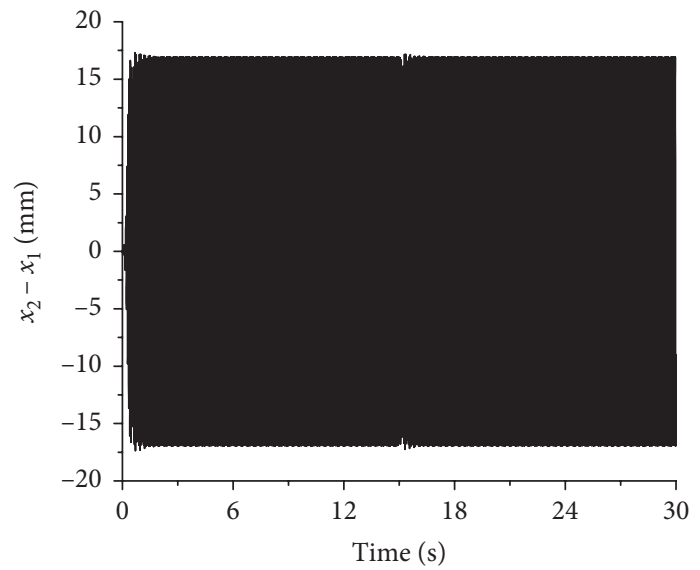

(e)

FIGURE 7: Numerical results in the subresonant case in regard of $\omega_{0}$ (or $\omega_{\text {Inv }}$ ): (a) rotational speed of the two motors, (b) phase difference between exciters 1 and 2, (c) response of rigid body $m_{1}$ in the $x$-direction, (d) response of rigid body $m_{2}$ in $x$-direction, and (e) difference of responses of two rigid bodies in the $x$-direction $\left(x_{2}-x_{1}=x_{12}\right)$.

from $A_{12}$, and the stable phase difference is $2 \alpha$. When the stable phase difference is close to zero, namely, $0.5365<z<1$ or $\omega_{1}^{\prime}<\omega_{m 0}<\omega_{\text {Inv }}, \lambda_{12}$ increases with the increase in frequency ratio $z$. At the resonant point, $\lambda_{12}$ reaches a maximum. When $z>1$, the stable phase difference between two rigid bodies is stabilized in the vicinity of $\pi$, and two 




\section{——- Motor 1}

\section{—— Motor 2}

(a)

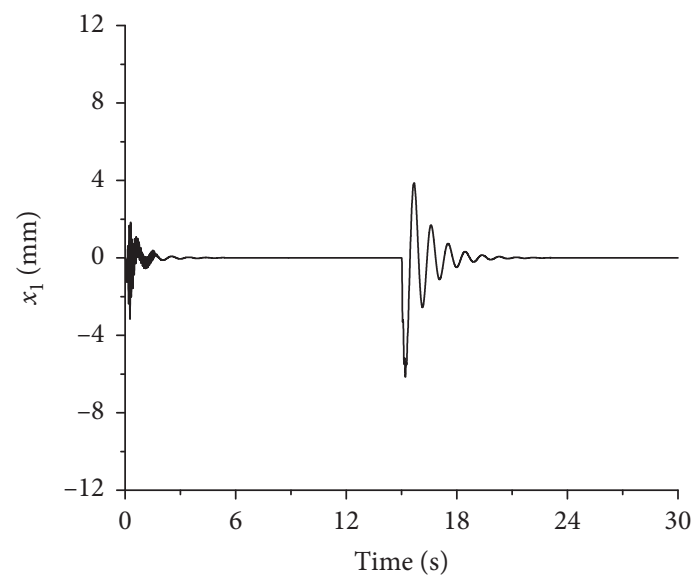

(c)

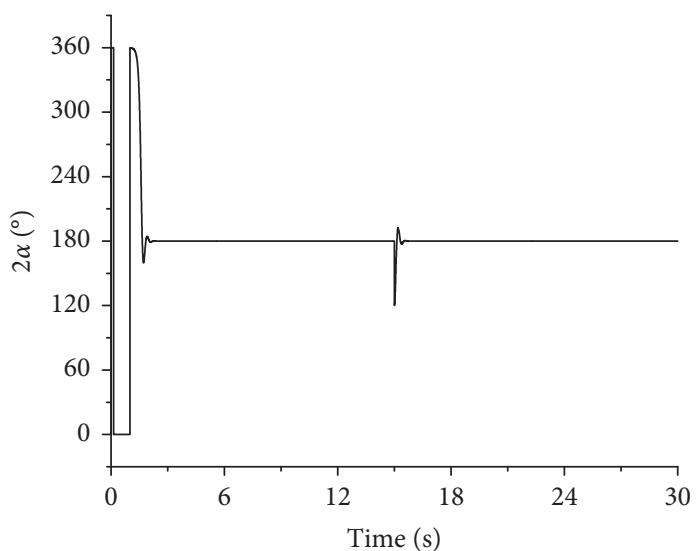

(b)

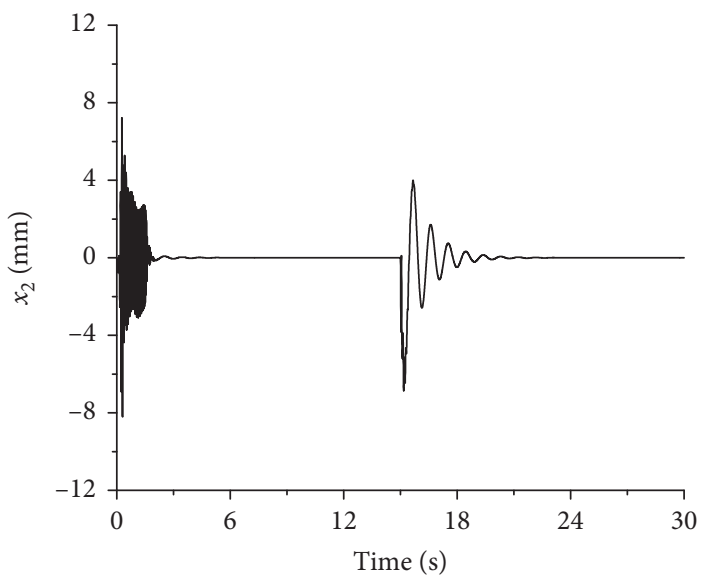

(d)



(e)

FIGURE 8: Numerical results in the super-resonant case in regard of $\omega_{0}$ (or $\left.\omega_{\text {Inv }}\right)$ : (a) rotational speed of the two motors, (b) phase difference between exciters 1 and 2, (c) response of rigid body $m_{1}$ in the $x$-direction, (d) response of rigid body $\mathrm{m}_{2}$ in the $x$-direction, and (e) difference of responses of two rigid bodies in the $x$-direction $\left(x_{2}-x_{1}=x_{12}\right)$. 


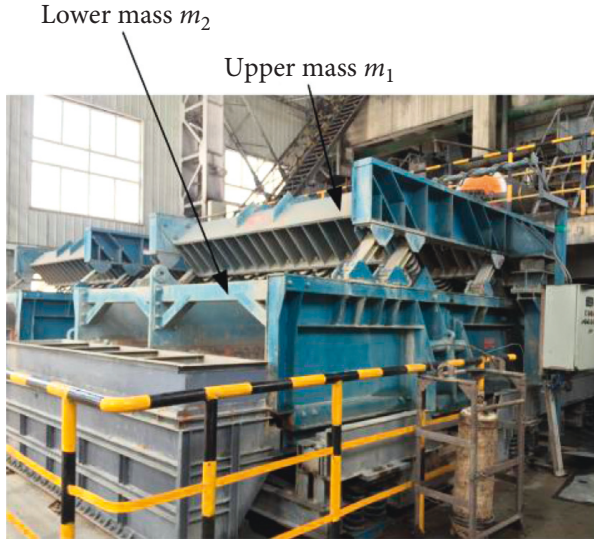

(a)

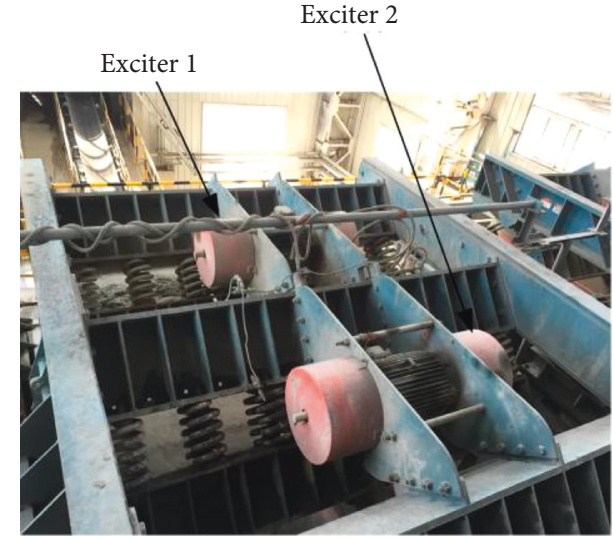

(b)

FIGURE 9: Vibrating machine with the dual-mass system driven by two exciters. (a) Front view. (b) Top view.

exciting forces provide $d$ by two motors cancel each other. The value of $\lambda_{12}$ is close to zero.

Based on the facts given above, reasonable working points of the system should be selected in the subresonant or near-subresonant region. This ensures relatively large and stable amplitude of relative motion.

\section{Simulation Analysis of Two Resonant Cases Based on Theoretical Results}

5.1. Simulation Results in the Subresonant Case regarding $\omega_{0}$. Figure 7 shows that the vibrating system operates in the subresonant region regarding $\omega_{\text {Inv }}\left(\right.$ or $\left.\omega_{0}\right)$. Under this condition, during starting, stable values are obtained at the same time because the two exciters are the same. At this time, because the system satisfies the coupling effect and stability criterion, both the exciters synchronize and quickly stabilize.

Figure 7(a) shows that, in the steady state, the synchronous operating frequency is $\omega_{m 0} \approx 650 \mathrm{r} \cdot \mathrm{min}^{-1}$, which is smaller than $\omega_{\text {Inv }} \approx \omega_{0}\left(\omega_{\text {Inv }} \approx \omega_{0}=712 \mathrm{r} \cdot \mathrm{min}^{-1}\right)$ and far greater than $\omega_{\mathrm{Sa}}$. This fact can be observed in Figure 7(e). There is enough large and stable amplitude of relative motion with the inverse phases in the $x$-direction. The phase difference between exciters 1 and 2 is close to zero, as shown in Figure $7(\mathrm{~b})$. The rigid body $m_{2}$ has an ideal amplitude in the $x$-direction, as shown in Figure $7(\mathrm{~d})$. All of these are engineering aspirations; therefore, the subresonant state of the system regarding $\omega_{0}$ can be applied to engineering.

5.2. Simulation Results in the Super-Resonant Case regarding $\omega_{0}$. Figure 8 shows that the synchronous operating frequency is close to $971 \mathrm{r} \cdot \mathrm{min}^{-1}$, and the stable phase difference is close to $\pi$. The amplitude of relative motion with the inverse phases in the $x$-direction is close to zero, as shown in Figure 8(e). None of the above can satisfy engineering applications; therefore, the super-resonant state of the system regarding $\omega_{0}$ cannot be applied to engineering.
TABle 1: Parameters of the vibrating screen.

\begin{tabular}{lc}
\hline Parameters & Value \\
\hline Mass of the rigid body $m_{1},(\mathrm{~kg})$ & 4000 \\
Mass of the rigid body $m_{2},(\mathrm{~kg})$ & 17000 \\
Stiffness of the spring between $m_{1}$ and $m_{2}, k_{1 x},(\mathrm{~N} / \mathrm{m})$ & 12000000 \\
Stiffness of the spring between foundation and $m_{2}$, & 400000 \\
$k_{2 x},(\mathrm{~N} / \mathrm{m})$ & \\
Damping coefficient between $m_{1}$ and $m_{2}$, & 235.2 \\
$f_{1 x},(\mathrm{Ns} / \mathrm{m})$ & \\
Damping coefficient between foundation and $m_{2}$, & 227.1 \\
$f_{2 x},(\mathrm{Ns} / \mathrm{m})$ & 3.2 \\
Mass of two exciters $m_{0},(\mathrm{~kg})$ & 0.08 \\
Eccentric radius of two exciters, $r,(\mathrm{~m})$ & 0.12 \\
Working amplitude of rigid body $m_{2}, \lambda_{2},(\mathrm{~m})$ & 172 \\
Phase relationship between two rigid bodies & \\
$\gamma_{1 x}-\gamma_{2 x},\left(^{\circ}\right)$ &
\end{tabular}

TABle 2: Parameters of two exciters.

\begin{tabular}{lcc}
\hline Parameters & Exciter 1 & Exciter 2 \\
\hline Rated frequency $f_{0},(\mathrm{~Hz})$ & 50 & 50 \\
Rated voltage $U_{n},(\mathrm{~V})$ & 380 & 380 \\
Rated speed $(\mathrm{r} / \mathrm{min})$ & 780 & 780 \\
Damping coefficients $f_{01}, f_{02},(\mathrm{Nm} / \mathrm{s})$ & 0.008 & 0.008 \\
\hline
\end{tabular}

\section{Engineering Application of the Theory of Synchronization of a Dual-Mass Vibrating System with Two Exciters}

The synchronization theory and dynamic model of the dualmass vibrating system with two exciters described in this paper were successfully applied to enterprise engineering. Figure 9 shows the engineering application of the dynamic model shown in Figure 1. Two eccentric exciters are symmetrically mounted on the upper mass $m_{1}$, as shown in Figure 9(b). The material is screened on the lower mass $m_{2}$, as shown in Figure 9(a).

The vibrating machine with the dual-mass system driven by two exciters is a vibrating screen. Tables 1 and 2 show the 
parameters of vibrating screen and parameters of two exciters of the vibrating screen separator as shown in Figure 9, respectively.

In practical engineering applications, two identical exciters are usually selected to generate more stable exciting forces. According to dynamic characteristics such as the synchronization criterion and stability criterion, as well as amplitude-frequency response, the structure of the system is optimized to match the parameters so that the machine can achieve the desired effect. Among them, the key problem lies in the selection of reasonable working points of the system.

Based on the effect of the material medium on vibrating machinery and combined with engineering experience, in the engineering example, the best working point can be selected in the range of $z=\omega_{m 0} / \omega_{0}=0.85 \sim 0.97$.

The structure of the system is compact and relatively simple. Using the principle of synchronization, many mandatory synchronizations of maintenance trouble can be removed and satisfy the needs of engineering. The market prospect is considerable.

\section{Conclusions}

The synchronization and stability criterion of two exciters were obtained. The coefficient of synchronization ability was used to identify the strength of synchronization between two exciters. Similarly, the coefficient of the ability of stability was used to determine its stability strength. In engineering, the structural parameters of a system can be optimized and matched according to the theory and numerical results, and the coefficient of synchronization ability of the system is greater than zero, which can be obtained. It can be ensured that the phase difference of the systems' subresonant or near-subresonant regions is stabilized around 0 . Importantly, the reasonable working points of the system can be selected in relative motion with the reverse phases between two rigid bodies to satisfy the needs of engineering.

The numerical results show that the system satisfies the engineering requirements under the subresonant state, while the super-resonant state cannot satisfy the engineering requirements.

Base on some theoretical and numerical analyses, the ideal working points of the total system can be selected in the subresonant or near-subresonant states.

It can be observed from the engineering application that the best working point should be selected in the range of $z=\omega_{m 0} / \omega_{0}=0.85 \sim 0.97$.

In engineering, the design of vibrating equipment can be guided by the theory of subresonant synchronization, such as subresonant approximately linear self-synchronous vibrating centrifuge, self-synchronous vibrating conveyor, and nonlinear self-synchronous vibrating centrifuge.

\section{Data Availability}

The data used to support the findings of this study are available from the corresponding author upon request.

\section{Conflicts of Interest}

The authors declare no conflicts of interest with respect to the research and/or publication of this article.

\section{Acknowledgments}

This project was supported by the National Natural Science Foundation of China (51675090 and 51705337).

\section{References}

[1] A. Pikovsky, M. Rosenblum, J. Kuths et al., Synchronization: A Universal Concept in Nonlinear Sciences, Cambridge University Press, Cambridge, UK, 2003.

[2] H. Junichi, I. Daisuke, M. Ichiro, and K. Takao, "Proposal of encoder-less time synchronous averaging method utilizing nonlinear oscillator for gears in operation," Journal of Advanced Mechanical Design, Systems, and Manufacturing, vol. 12, no. 2, Article ID JAMDSM0039, 2018.

[3] R. Yamapi and P. Woafo, "Dynamics and synchronization of coupled self-sustained electromechanical devices," Journal of Sound and Vibration, vol. 285, no. 4-5, pp. 1151-1170, 2005.

[4] C. Huygens, Horologium Oscilatorium, Apud F. Muguet, Paris, France, 1673.

[5] I. I. Blekhman, Synchronization in Science and Technology, ASME Press, New York, NY, USA, 1988.

[6] I. I. Blekhman, Vibrational Mechanics, World Scientific Press, Singapore, 2000.

[7] I. I. Blekhman and N. P. Yaroshevich, "Extension of the domain of applicability of the integral stability criterion (extremum property) in synchronization problems," Journal of Applied Mathematics and Mechanics, vol. 68, no. 6, pp. 839-846, 2004.

[8] B. C. Wen, H. Zhang, S. Y. Liu, Q. He, and C. Y. Zhao, Theory and Techniques of Vibrating Machinery and Their Applications, Science Press, Beijing, China, 2010.

[9] B. C. Wen, J. Fan, C. Y. Zhao et al., Vibration Synchronization and Controlled Synchronization in Engineering, Science Press, Beijing, China, 2009.

[10] V. I. Babitsky and V. I. Krupenin, Vibration of Strongly Nonlinear Discontinuous Systems, Springer-Verlag, Berlin, Germany, 2001.

[11] J. Inoue, "On the self-synchronization of mechanical vibrators: part 5, shock vibrator," Transactions of the Japan Society of Mechanical Engineers, vol. 42, no. 353, pp. 103-110, 1976.

[12] J. Inoue, S. Miyaura, and O. Matsushita, "On the self-synchronization of mechanical vibrators: Part 3, forced frictional vibrations," Bulletin of JSME, vol. 35, pp. 1242-1248, 2008.

[13] C. Y. Zhao, Y. M. Zhang, and B. C. Wen, "Synchronization and general dynamic symmetry of a vibrating system with two exciters rotating in opposite directions," Chinese Physics B, vol. 19, no. 3, 2010.

[14] X. Zhang, B. Wen, and C. Zhao, "Synchronization of three non-identical coupled exciters with the same rotating directions in a far-resonant vibrating system," Journal of Sound and Vibration, vol. 332, no. 9, pp. 2300-2317, 2013.

[15] X. L. Zhang, C. Li, Z. H. Wang, and S. J. Cui, "Synchronous stability of four homodromy vibrators in a vibrating system with double resonant types," Shock and Vibration, vol. 2018, Article ID 9641231, 20 pages, 2018.

[16] X. Zhang, B. Wen, and C. Zhao, "Vibratory synchronization transmission of a cylindrical roller in a vibrating mechanical 
system excited by two exciters," Mechanical Systems and Signal Processing, vol. 96, pp. 88-103, 2017.

[17] J. Balthazar, J. Felix, and R. Brasil, "Short comments on selfsynchronization of two non-ideal sources supported by a flexible portal frame structure," JVC/Journal of Vibration and Control, vol. 10, no. 6, pp. 1739-1748, 2004.

[18] J. M. Balthazar, J. L. P. Felix, and R. M. Brasil, "Some comments on the numerical simulation of self-synchronization of four non-ideal exciters," Applied Mathematics and Computation, vol. 164, no. 2, pp. 615-625, 2005.

[19] H. Nijmeijer and R. Alejandro, "Synchronization of mechanical systems," World Scientific Series on Nonlinear Science Series A, vol. 46, 2003.

[20] X. Kong, X. Chen, J. Dou, X. Zhang, and B. Wen, "Controlled synchronization of two nonidentical homodromy coupling exciters driven by inductor motors in a vibratory system," Proceedings of the Institution of Mechanical Engineers, Part C: Journal of Mechanical Engineering Science, vol. 230, no. 17, pp. 3040-3054, 2016.

[21] A. Guzman, Derivatives and Integrals of Multivariable Functions, Springer, New York, NY, USA, 2003. 


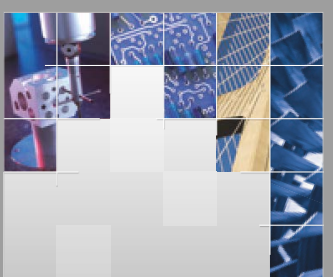

\section{Enfincering}
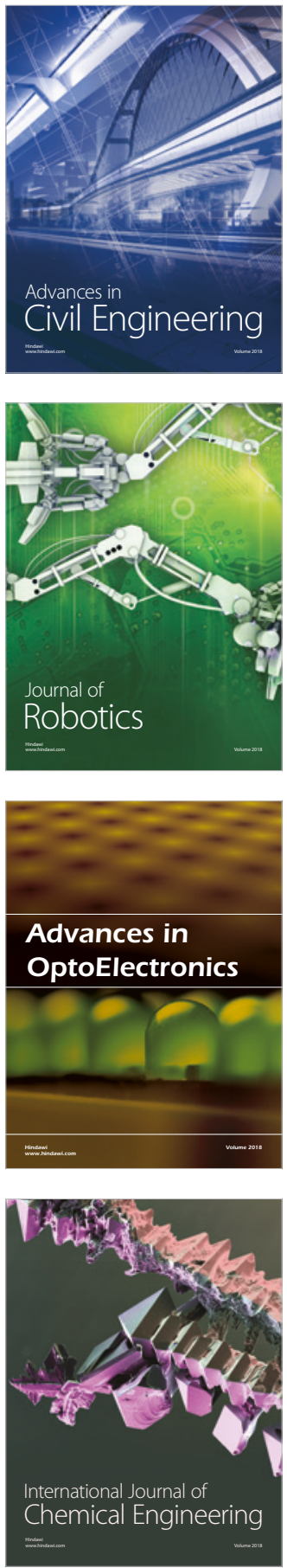

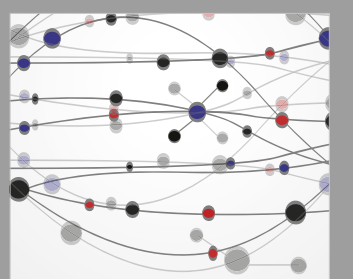

\section{Rotating \\ Machinery}

The Scientific World Journal

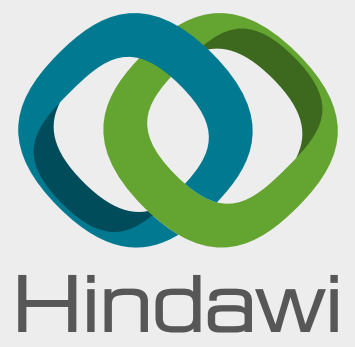

Submit your manuscripts at

www.hindawi.com
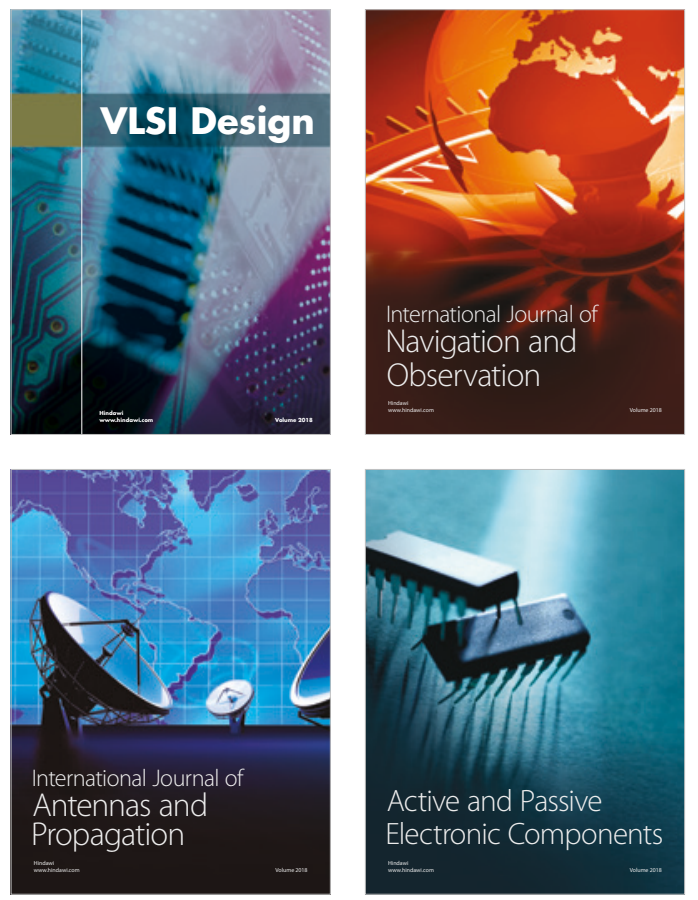
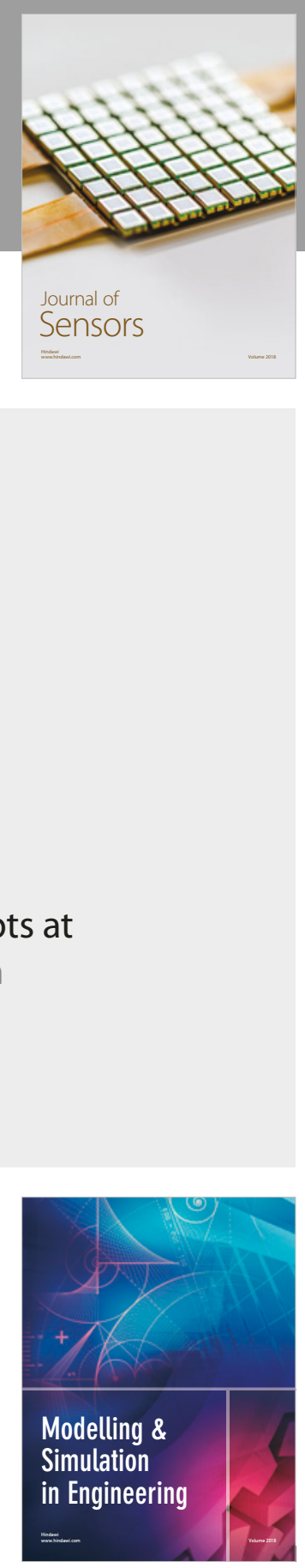

\section{Advances \\ Multimedia}
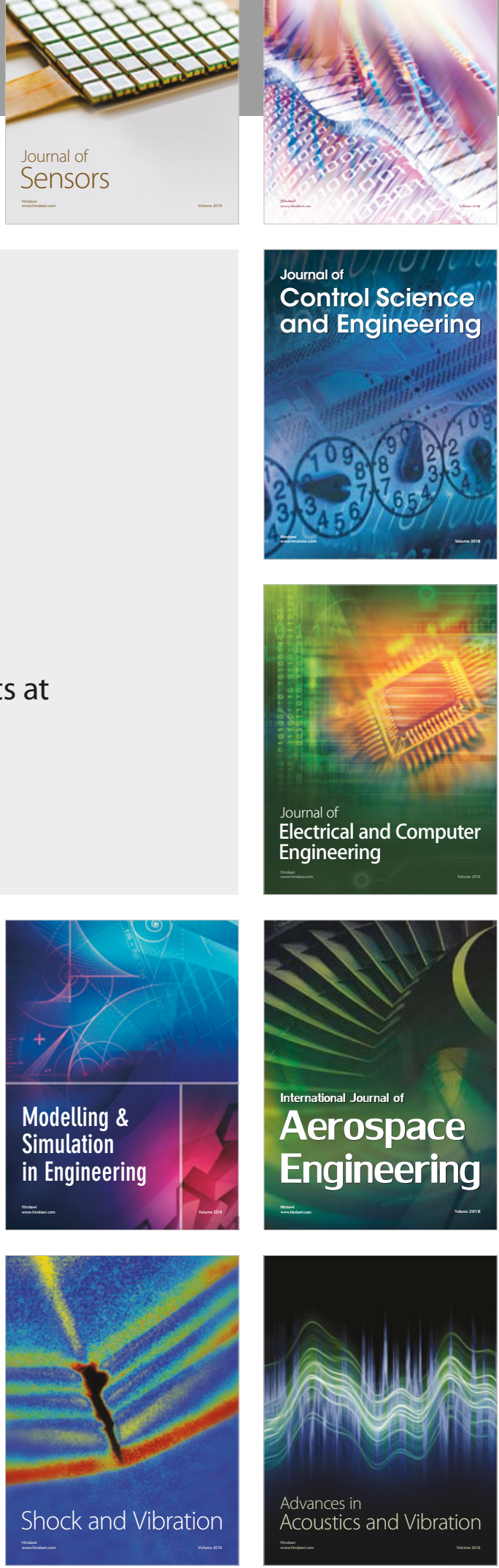